\title{
Tibial Plateau Leveling Osteotomy for Cranial Cruciate Ligament Rupture in Canines: Patient Selection and Reported Outcomes
}

This article was published in the following Dove Press journal:

Veterinary Medicine: Research and Reports

\author{
Andy Nanda (D) \\ Eric C Hans ${ }^{2}$ \\ 'Department of Surgery, IronHorse \\ VetCare, Dublin, CA, USA; ${ }^{2}$ Department \\ of Surgery, MedVet Indianapolis, \\ Indianapolis, IN, USA
}

\begin{abstract}
Cranial cruciate ligament (CCL) rupture is one of the most common causes of pelvic limb lameness in dogs. In a CCL deficient stifle, joint lameness develops from cranial tibial translation in relation to the distal femoral condyles. The tibial plateau leveling osteotomy (TPLO) is a well-described surgical procedure that neutralizes tibiofemoral shear force by altering the tibial plateau via a proximal tibial osteotomy, and subsequently restores normal limb function. Current literature for the TPLO was reviewed and used to describe the optimal patient selection and to report clinical outcome with the TPLO in comparison to other surgical options for CCL rupture. The clinical outcomes reported to date, particularly those utilizing objective outcome data, support the TPLO as being able to consistently return dogs to normal limb function with a low risk for long-term morbidity. The TPLO procedure was found to be widely applicable to dogs ranging from small breed to large and giant breed, and does not appear to have some of the limitations that exist with alternative surgical options. This review concludes that the TPLO is an excellent treatment option for any dog with CCL rupture due to the excellent long-term outcome and high rate of owner satisfaction previously reported. Despite these conclusions, it is important to remember that treatment selection for CCL rupture remains dependent on multiple considerations including patient factors, surgeon experience, and costs.
\end{abstract}

Keywords: TPLO, CCL, canine stifle, lateral suture, TTA

\section{Introduction}

The cranial cruciate ligament (CCL) is the primary stabilizer of the canine stifle joint. Loss of a functional CCL results in destabilization of the stifle joint with cranial tibial translation in relation to the distal femoral condyles during weightbearing and subsequent osteoarthritis formation. Rupture of the CCL is a common cause of non-traumatic pelvic limb lameness in dogs. ${ }^{1}$ This occurs most frequently through progressive degenerative rupture (i.e. cranial cruciate ligament disease); although, acute traumatic rupture is also described. The etiopathogenesis of CCL rupture is not fully understood, with various genetic, environmental, and mechanical factors influencing disease progression. ${ }^{2-6}$ Stifle joint inflammation or synovitis appears to be the initial clinical finding preceding complete CCL rupture. ${ }^{7,8}$ Once clinically detectable inflammation is present, subsequent rupture occurs in $85 \%$ of dogs. ${ }^{9}$ Treatment of choice for a CCL rupture is surgery to neutralize tibiofemoral shear force and re-establish active stifle joint stability during limb use. A variety of static and dynamic stifle joint procedures have been previously described, with the
Correspondence: Eric C Hans Indianapolis, 9650 Mayflower Park Dr

Carmel, IN 46032, USA

Tel + I 3178728387

$\mathrm{Fax}+$ I 3178721964

Email ehans.dvm@gmail.com 
tibial plateau leveling osteotomy (TPLO) emerging as the preferred treatment option for present-day surgeons. ${ }^{10}$ This is due to the TPLO demonstrating exceptional and repeatable clinical outcomes when compared to other surgical options.

\section{Development and Biomechanics of the TPLO}

The CCL ligament is the principle restraint against cranial tibial translation in relation to the distal femoral condyles. In 1993, Slocum proposed the "active model" of the stifle joint, which explains that the magnitude of the tibiofemoral shear force is dependent on the magnitude of the joint compressive force and the tibial plateau angle (TPA). ${ }^{11}$ In the normal stifle joint, this shear force is opposed by active elements, such as stifle joint flexor muscles, and by passive elements, including the CCL and the medial meniscus. In the CCL deficient stifle joint, cranial tibial translation in relation to the distal femoral condyles occurs during the stance phase of gait as the flexors' of the stifle joint alone are unable to neutralize the tibiofemoral shear force generated during stifle joint compression. Reducing the TPA in this model eliminates cranial tibial translation in relation to the distal femoral condyles by resolving the tibiofemoral shear force into the joint compressive force during stance, while also enhancing the flexors' ability to neutralize any residual shear force. Slocum utilized a radial osteotomy centered on the tibial intercondylar eminence to rotate the tibial plateau and reduce the TPA to $5^{\circ}$. A procedurespecific bone plate was then used to maintain tibial plateau positioning throughout osteotomy healing. Following successful initial clinical outcome, Slocum taught the TPLO procedure to fellow surgeons via courses. An in vitro study later concurred that neutralization of tibiofemoral shear force occurs at $6.5^{\circ} .{ }^{12}$ Clinically, the ideal TPA likely has mild variability dog to dog. Good clinical outcome has been reported following TPLO with postoperative TPA ranging from $0^{\circ}$ to $14^{\circ} .{ }^{13}$ That said, the majority of surgeons performing the TPLO plan osteotomy rotation utilizing a chart with an intended postoperative TPA of $5^{\circ}$, and rotation to within several degrees of this intended postoperative TPA value is considered critical to successful outcome. There is a caution to avoid significant overrotation of the TPA as this may lead to excessive stress on the caudal cruciate ligament and predispose to caudal cruciate ligament injury. ${ }^{12}$

\section{Advances of the TPLO Procedure}

The TPLO has been the most researched orthopedic procedure in veterinary medicine over the past 20 years. As the number of surgeons regularly performing TPLO surgery has increased, numerous publications have been generated to refine the procedure by identifying and decreasing complications, improving osteotomy planning and efficiency, and detailing advantages offered by newer implant technology. These refinements have resulted in dogs of any size with CCL rupture being appropriate candidates for the TPLO surgery.

One of the more important transitions over the past 10 years has been the replacement of the traditional compression based TPLO plate and screw constructs to precontoured locking plate and screw constructs. Traditional non-locking bone plates rely on the friction between the bone and the plate generated by screw torque to stabilize the osteotomy. ${ }^{14}$ This is more challenging to achieve in the softer cancellous bone of the proximal tibial. An early complication of the TPLO was secondary loss of reduction, or "rock back", in which the TPA would shift significantly between that achieved immediately postoperative and that measured at radiographic followup. ${ }^{15}$ This change can affect long-term outcome by failing to fully neutralize tibiofemoral shear force, as well as predispose to fracture or other major complication. Locking plates and screws function as a single beam construct, in which each screw locks into the bone plate and the strength of the construct is equal to the sum of all the bone-screw interfaces. ${ }^{14}$ This prohibits the typical mode of failure in non-locking plates, which is toggling or loosening of screws, and subsequent loss of bone stability. Locking plate-screw constructs are particularly advantageous in softer cancellous bone where maintaining high frictional forces can be more challenging. This has been proven in clinical and mechanical studies where initial TPA is better maintained using locking plates. ${ }^{16-18}$ Locking bone plates also offer other advantages such as a larger core diameter to better resist bending forces, better preservation of the periosteal blood supply, and angled screw holes to minimize the risk of intra-articular screw placement. ${ }^{19-21}$

Micromotion at the osteotomy, even without significant loss of reduction, has also been implicated in increased rates of surgical site infection, particularly within populations of large and giant breed dogs. Several reports have documented increased rates of surgical site infection in 
large and giant breed dogs using traditional non-locking bone plates. ${ }^{22,23}$ Locking bone plates appear to be protective against surgical site infection in this subpopulation of dogs due to superior mechanical stability and biologic preservation. ${ }^{23}$ Locking TPLO plates are now readily commercially available through numerous implant manufacturers in screw sizes ranging from $2.0 \mathrm{~mm}$ through $3.5 \mathrm{~mm}$. Broad and jumbo $3.5 \mathrm{~mm}$ TPLO plates also exist to accommodate giant breeds and provide optimal osteotomy stabilization.

Refined execution of the TPLO has also improved the efficiency of the procedure and reduced clinical complication. Computer software is now commonplace in veterinary specialty centers which allow for osteotomy planning via templating the size of radial saw blade, as well as making reference points to best center the osteotomy on the tibial intercondylar eminence. Failure to center the osteotomy appropriately can result in a long-axis drift, and prohibit achieving the desired postoperative TPA. ${ }^{24}$ Pre-operative TPLO planning is now advocated by most surgeons to achieve consistent rotation which is critical to optimizing clinical outcome. Furthermore, failure to center the osteotomy can predispose the patient to fracture of the tibial tuberosity. General recommendations are to maintain a tibial tuberosity width of $1 \mathrm{~cm}$, as well as ensuring the narrowest aspect of the tuberosity does not occur distal to the insertion of the patellar ligament. ${ }^{25,26}$ Even antirotational pin placement has been evaluated with recommendations for placement proximal to the patellar ligament insertion to reduce the risk for complication. ${ }^{26}$

This advanced understanding of surgical technique and execution, combined with improving implant technology, has made the TPLO one of the safest and most repeatable elective orthopedic procedures to date. While the risk for major complication cannot be completely eliminated in any procedure, their reduction with the TPLO over the past 20 years has been the result of the hard work of numerous clinicians and scientists in the veterinary surgery community.

\section{Clinical Outcome with TPLO}

The TPLO is currently the most commonly recommended procedure by veterinary surgeons for the treatment of CCL rupture. ${ }^{10,27}$ That said, several other procedures exist and may be alternatively selected for the treatment of CCL rupture. The TPLO is a dynamic osteotomy-based procedure that neutralizes tibiofemoral shear force during weight bearing. An alternative dynamic osteotomy-based procedure that is commonly compared to the TPLO is the tibial tuberosity advancement (TTA). The TTA procedure has a competing biomechanical model in that tibiofemoral shear force is oriented parallel to the patellar ligament, instead of perpendicular to the TPA, and that by advancing the patellar ligament insertion to a $90^{\circ}$ orientation to the TPA, a crossover point will occur in which tibiofemoral shear force is neutralized. ${ }^{28}$ Successful clinical outcome has been described in a large number of patients following TTA surgery and is the primary alternative procedure to the TPLO for medium and large breed dogs. ${ }^{29-31}$ Static stifle joint stabilization procedures are also available for the treatment of CCL rupture with the extracapsular lateral suture stabilization (ELSS) being the most common. The ELSS procedure utilizes a monofilament nylon material to constrain the stifle joint by prohibiting cranial tibial thrust similar to an intact CCL. ${ }^{32}$

Each of the surgical options listed above has demonstrated initial improvement in pain and lameness postoperatively. The ideal surgical option though should consistently return the patient to normal or near-normal limb function long term with little risk for patient morbidity. With that in mind, direct comparison between procedures can be difficult as many studies are comprised of subjective outcome measures, lack standardization, and have variation in study duration. This was highlighted when a review of CCL treatment options in 2005 was unable to conclude that any surgical option was superior to the other, or if any of these treatment options had the ability to return the patient to pre-injury function. ${ }^{33}$ As a result, there have been efforts to better utilize evidencebased medicine with objective data and long-term outcome reporting in veterinary orthopedics. Direct clinical comparison of treatment options has primarily focused on comparing the TPLO, TTA, and ELSS to one another given their commonality within the surgical community.

Force-platform data represents an objective, unbiased outcome measure for direct comparison of limb function following surgery. Several studies have utilized forceplatform data to compare the clinical outcome between TPLO and the ELSS and TTA procedures. Gordon-Evans et al reported significantly higher peak vertical force for TPLO when compared to ELSS at 1 year following surgery, as well as greater client satisfaction with the TPLO procedure overall (93\% with TPLO vs $73 \%$ with ELSS). ${ }^{34}$ These results were corroborated in a separate study where TPLO again demonstrated significantly greater limb function following surgery when compared to ELSS using 
force platform data. In addition, this study also compared outcome following TPLO to normal dogs without CCL disease and concluded that TPLO resulted in limb function that was indistinguishable from the control normal dog population at 1 year following surgery. ${ }^{35}$ The TTA procedure was evaluated in a similar manner by this same research group. Conclusions for the TTA were that at a walking pace it appeared to achieve normal limb function; but, when evaluated at a trotting pace dogs treated by TTA were found to have inferior function to those treated with TPLO. ${ }^{36}$ Trotting gaits are more sensitive for detecting mild lameness. ${ }^{37}$

Another significant factor influencing the long-term outcome is the progression of osteoarthritis following surgery. Osteoarthritis progression has been documented following TPLO, TTA, and ELSS in multiple studies. ${ }^{38-40}$ Significant osteoarthritis progression can limit successful long-term outcome due to chronic stifle joint pain. Recently, a study was published that compared the long term ( $>3$ years) progression of osteoarthritis following both TPLO and TTA. ${ }^{40}$ Radiographic osteoarthritis progression was also correlated with clinical signs using the Canine Brief Pain Inventory questionnaire. In that study, osteoarthritis progressed more significantly after TTA surgery and dogs treated with TPLO had less pain and mobility issues as assessed by their owners. ${ }^{40}$

The summary of these studies finds the TPLO to be the ideal surgery for returning patients to optimal long-term function. Another systematic review of the literature for the treatment of CCL was published in 2014. Unlike the 2005 publication, this paper effectively concluded that the available evidence supported the TPLO as the surgery most likely to return dogs to normal limb function. ${ }^{41}$

\section{TPLO Patient Selection}

The TPLO procedure is most commonly described in medium to large breed dogs that are overrepresented with CCL rupture, such as Labradors, Golden Retrievers, and Rottweilers. The excellent outcomes described following the TPLO procedure have caused many surgeons to advocate for this procedure for any dog with CCL rupture regardless of patient age or size. A variety of implant sizes are now available, as well as radial saw blades ranging from $12 \mathrm{~mm}$ to $30 \mathrm{~mm}$ to accommodate a wide patient selection. Multiple reports have been published recently demonstrating a low complication rate and ability to adequately perform TPLO surgery in smaller breed dogs. ${ }^{42-44}$ These dogs can be challenging as some are predisposed to having higher or "excessive" TPAs. ${ }^{42,43}$ The TPLO procedure is considered a more ideal treatment option for dogs with high TPA. It has been cautioned against using the TTA procedure in dogs with high TPA as it may be more difficult to neutralize tibiofemoral shear force. ${ }^{45}$ Likewise, giant breed dogs are also good TPLO candidates, and in some cases may only be able to receive TPLO surgery as procedures such as the TTA can be limited by available implants and the ability to achieve adequate tuberosity advancement. ${ }^{45,46}$ As previously stated though, complication rates can be higher in these patients so clients should be educated about the importance of adherence to postoperative activity restriction and surgeons should utilize stable locking constructs. ${ }^{21,22,47}$

In addition to patient size, owner expectations of performance are also important. The TPLO offers the best prognosis for return to full athletic function. Working and agility dogs may benefit the most from this procedure in allowing them to return to pre-injury function. A study of agility dogs demonstrated a good prognosis for return to competition following TPLO surgery. ${ }^{48}$ Many of these dogs can vary in size and having a procedure that best optimizes outcome that is universally applicable is crucial to handlers and owners of dogs with specific function.

A subset of dogs will present with bilateral CCL rupture. These dogs can be particularly debilitated with regard to basic mobility, and in some instances can even be misdiagnosed for neurologic disorders. Traditionally, dogs with bilateral CCL rupture have been treated by TPLO procedures staged 4-8 weeks apart. An alternative option is to perform single-session bilateral TPLO surgery. There are conflicting reports as to if single-session bilateral TPLO surgery carries a greater risk for postoperative complication compared to unilateral procedures. ${ }^{25,47,49-51}$ It is difficult to compare complications in these prior reports given the variability of implants utilized and the evolving improvements in surgical technique. Presently, one author $(\mathrm{ECH})$ of this report regularly performs singlesession bilateral TPLO with strict patient selection criteria (i.e. dogs $<100 \mathrm{lbs}$, appropriate body condition, compliant owners) and has anecdotally not experienced an increased rate of complication. Owner satisfaction is also high with single-session bilateral surgery in our experience as it offers the benefit of a singular recovery period and an earlier return to function compared to staged surgery, as well as financial savings to the owner. Performance of a single-session bilateral TPLO is dependent on patient and surgeon factors, but can be considered an acceptable 
option on a case by case basis and represents another advantage of the TPLO procedure in being able to address these significantly debilitated patients.

In addition to anticipated clinical outcome and individual patient factors, cost considerations can also influence owners in electing to perform TPLO surgery in comparison to other treatment options. TPLO surgery is typically performed by board-certified veterinary surgeons and can have substantial cost due to specialized instrumentation, implants, and required perioperative care at specialty surgical centers. That said, TTA and other non-TPLO osteotomy-based procedures are similarly priced as they too are commonly performed at specialty surgical centers. Extracapsular lateral suture stabilization is more cost-effective and can be performed with a low risk of failure in small dogs. As such, it is a common alternative that is elected in patients $<15 \mathrm{~kg}$. This procedure can also be offered by general practitioners due to the lack of required specialized equipment. As started earlier though, ELSS is not considered an appropriate option for larger dogs as suture line failure can occur leading to the unacceptable outcome and low client satisfaction. It is not uncommon for TPLO to be recommended as a revision option in larger dogs with persistent instability following the failure of an ELSS procedure.

\section{Conclusion}

The culmination of clinical evidence has evolved the TPLO from a "new procedure" to the present-day standard of care for the CCL deficient stifle joint. The demands of surgeons and pet owners to offer the best return to function have led to accommodating patients of all sizes with this procedure. That said, treatment selection for CCL rupture is dependent on multiple considerations beyond clinical outcome, such as patient factors, surgeon experience, and costs. Further research efforts will likely find additional ways to maximize the clinical outcome in dogs with CCL disease; however, until another procedure is proven to return dogs to normal function with a low rate of complication, the TPLO will remain a common procedure in veterinary orthopedics.

\section{Disclosure}

The authors report no conflicts of interest in this work.

\section{References}

1. Witsberger TH, Villamil JA, Schultz LG, Hahn AW, Cook JL. Prevalence of and risk factors for hip dysplasia and cranial cruciate ligament deficiency in dogs. $J$ Am Vet Med Assoc. 2008;232 (12):1818-1824. doi:10.2460/javma.232.12.1818
2. Ichinohe T, Kanno N, Harada Y, Yogo T, Tagawa M, Hara Y. Histological and immunohistological analysis of degenerative changes in the cranial cruciate ligament in a canine model of excessive tibial plateau angle. Vet Comp Orthop Traumatol. 2015;28 (4):240-249. doi:10.3415/VCOT-14-08-0128

3. Baker LA, Kirkpatrick B, Rosa GJM, et al. Genome-wide association analysis in dogs implicates 99 loci as risk variants for anterior cruciate ligament rupture. PLoS One. 2017;12(4):e0173810. doi:10.1371/journal.pone.0173810

4. Wilke VL, Zhang S, Evans RB, Conzemius MG, Rothschild MF. Identification of chromosomal regions associated with cranial cruciate ligament rupture in a population of Newfoundlands. Am J Vet Res. 2009;70(8):1013-1017. doi:10.2460/ajvr.70.8.1013

5. Ragetly CA, Evans R, Mostafa AA, Griffon DJ. Multivariate analysis of morphometric characteristics to evaluate risk factors for cranial cruciate ligament deficiency in Labrador retrievers. Vet Surg. 2011;40 (3):327-333. doi:10.1111/j.1532-950X.2010.00787.x

6. Duval JM, Budsberg SC, Flo GL, Sammarco JL. Breed, sex, and body weight as risk factors for rupture of the cranial cruciate ligament in young dogs. J Am Vet Med Assoc. 1999;215(6):811-814.

7. Bleedorn JA, Greuel EN, Manley PA, et al. Synovitis in dogs with stable stifle joints and incipient cranial cruciate ligament rupture: a cross-sectional study. Vet Surg. 2011;40(5):531-543. doi:10.1111/ j.1532-950X.2011.00841.x

8. Little JP, Bleedorn JA, Sutherland BJ, et al. Arthroscopic assessment of stifle synovitis in dogs with cranial cruciate ligament rupture. Rosenfeld CS. ed. PLoS One. 2014;9(6):e97329-12. doi:10.1371/ journal.pone.0097329.

9. Fuller MC, Hayashi K, Bruecker KA, et al. Evaluation of the radiographic infrapatellar fat pad sign of the contralateral stifle joint as a risk factor for subsequent contralateral cranial cruciate ligament rupture in dogs with unilateral rupture: 96 cases (2006-2007). $J$ Am Vet Med Assoc. 2014;244(3):328-338. doi:10.2460/javma.244.3.328

10. von DJF P, Kowaleski MP, Glassman M, Déjardin LM. Results of a survey of veterinary orthopedic society members on the preferred method for treating cranial cruciate ligament rupture in dogs weighing more than 15 kilograms (33 pounds). J Am Vet Med Assoc. 2018;253(5):586-597. doi:10.2460/javma.253.5.586

11. Slocum B, Slocum TD. Tibial plateau leveling osteotomy for repair of cranial cruciate ligament rupture in the canine. Vet Clin North Am Small Anim Pract. 1993;23:777-795. doi:10.1016/S0195-5616(93) 50082-7

12. Warzee CC, Dejardin LM, Arnoczky SP, Perry RL. Effect of tibial plateau leveling on cranial and caudal tibial thrusts in canine cranial cruciate-deficient stifles: an in vitro experimental study. Vet Surg. 2001;30(3):278-286. doi:10.1053/jvet.2001.21400

13. Robinson DA, Mason DR, Evans R, Conzemius MG. The effect of tibial plateau angle on ground reaction forces 4-17 months after tibial plateau leveling osteotomy in Labrador Retrievers. Vet Surg. 2006;35 (3):294-299. doi:10.1111/j.1532-950X.2006.00147.x

14. Biedrzycki AH. Dynamic compression vs. Locking plating - is One "Better"? A review of biomechanical principals and in vitro testing. In: Barnhart MD, Maritato KC, editors. Locking Plates in Veterinary Orthopedics. New Jersey: John Wiley \& Sons, Inc.; 2019:25-37.

15. Moeller EM, Cross AR, Rapoff AJ. Change in tibial plateau angle after tibial plateau leveling osteotomy in dogs. Vet Surg. 2006;35 (5):460-464. doi:10.1111/j.1532-950X.2006.00175.x

16. Conkling AL, Fagin B, Daye RM. Comparison of tibial plateau angle changes after tibial plateau leveling osteotomy fixation with conventional or locking screw technology. Vet Surg. 2010;39(4):475-481. doi:10.1111/j.1532-950X.2010.00656.x

17. Kloc PA, Kowaleski MP, Litsky AS, Brown NO, Johnson KA. Biomechanical comparison of two alternative tibial plateau leveling osteotomy plates with the original standard in an axially loaded gap model: an in vitro study. Vet Surg. 2009;38(1):40-48. doi:10.1111/ j.1532-950X.2008.00464.x 
18. Leitner M, Pearce SG, Windolf M, et al. Comparison of locking and conventional screws for maintenance of tibial plateau positioning and biomechanical stability after locking tibial plateau leveling osteotomy plate fixation. Vet Surg. 2008;37(4):357-365. doi:10.1111/j.1532950X.2008.00389.x

19. Kowaleski MP, Boudrieau RJ, Beale BS, Piras A, Hulse D, Johnson KA. Radiographic outcome and complications of tibial plateau leveling osteotomy stabilized with an anatomically contoured locking bone plate. Vet Surg. 2013;42(7):847-852. doi:10.1111/ j.1532-950X.2013.12048.x

20. Perren SM. Evolution of the internal fixation of long bone fractures. The scientific basis of biological internal fixation: choosing a new balance between stability and biology. J Bone Joint Surg Br. 2002;84 (8):1093-1110. doi:10.1302/0301-620X.84B8.0841093

21. Gautier E, Sommer C. Guidelines for the clinical application of the LCP. Injury. 2003;34:63-76. doi:10.1016/j.injury.2003.09.026

22. Hans EC, Barnhart MD, Kennedy SC, Naber SJ. Comparison of complications following tibial tuberosity advancement and tibial plateau levelling osteotomy in very large and giant dogs $50 \mathrm{~kg}$ or more in body weight. Vet Comp Orthop Traumatol. 2017;30(4):299-305. doi:10.3415/VCOT-16-07-0106

23. Solano MA, Danielski A, Kovach K, Fitzpatrick N, Farrell M. Locking plate and screw fixation after tibial plateau leveling osteotomy reduces postoperative infection rate in dogs over $50 \mathrm{~kg}$. Vet Surg. 2015;44(1):59-64. doi:10.1111/j.1532-950X.2014.12212.x

24. Kowaleski MP, Apelt D, Mattoon JS, Litsky AS. The effect of tibial plateau leveling osteotomy position on cranial tibial subluxation: an in vitro study. Vet Surg. 2005;34(4):332-336. doi:10.1111/j.1532950X.2005.00051.x

25. Bergh MS, Rajala-Schultz P, Johnson KA. Risk factors for tibial tuberosity fracture after tibial plateau leveling osteotomy in dogs. Vet Surg. 2008;37(4):374-382. doi:10.1111/j.1532-950X.2008.00391.x

26. Mehrkens LR, Hudson CC, Cole GL. Factors associated with early tibial tuberosity fracture after tibial plateau leveling osteotomy. Vet Surg. 2018;47(5):634-639. doi:10.1111/vsu.12915

27. Duerr FM, Martin KW, Rishniw M, Palmer RH, Selmic LE. Treatment of canine cranial cruciate ligament disease. Vet Comp Orthop Traumatol. 2014;27(6):478-483. doi:10.3415/VCOT-14-03-0047

28. Apelt D, Kowaleski MP, Boudrieau RJ. Effect of tibial tuberosity advancement on cranial tibial subluxation in canine cranial cruciate-deficient stifle joints: an in vitro experimental study. Vet Surg. 2007;36(2):170-177. doi:10.1111/j.1532-950X.2007.00250.x

29. Skinner OT, Kim SE, Lewis DD, Pozzi A. In vivo femorotibial subluxation during weight-bearing and clinical outcome following tibial tuberosity advancement for cranial cruciate ligament insufficiency in dogs. Vet J. 2013;196(1):86-91. doi:10.1016/j.tvj1.2012.08.003

30. Dymond NL, Goldsmid SE, Simpson DJ. Tibial tuberosity advancement in 92 canine stifles: initial results, clinical outcome and owner evaluation. Aust Vet J. 2010;88(10):381-385. doi:10.1111/j.17510813.2010.00627.x

31. Steinberg EJ, Prata RG, Palazzini K, Brown DC. Tibial tuberosity advancement for treatment of CrCL injury: complications and owner satisfaction. J Am Anim Hosp Assoc. 2011;47(4):250-257. doi:10.5326/JAAHA-MS-5574

32. Tonks CA, Lewis DD, Pozzi A. A review of extra-articular prosthetic stabilization of the cranial cruciate ligament-deficient stifle. Vet Comp Orthop Traumatol. 2011;24(3):167-177. doi:10.3415/VCOT-10-060084

33. Aragon CL, Budsberg SC. Applications of evidence-based medicine: cranial cruciate ligament injury repair in the dog. Vet Surg. 2005;34 (2):93-98. doi:10.1111/j.1532-950X.2005.00016.x

34. Gordon-Evans WJ, Griffon DJ, Bubb C, Knap KM, Sullivan M, Evans RB. Comparison of lateral fabellar suture and tibial plateau leveling osteotomy techniques for treatment of dogs with cranial cruciate ligament disease. $J$ Am Vet Med Assoc. 2013;243 (5):675-680. doi:10.2460/javma.243.5.675
35. Nelson SA, Krotscheck U, Rawlinson J, Todhunter RJ, Zhang Z, Mohammed H. Long-term functional outcome of tibial plateau leveling osteotomy versus extracapsular repair in a heterogeneous population of dogs. Vet Surg. 2013;42(1):38-50. doi:10.1111/j.1532-95 0X.2012.01052.x

36. Krotscheck U, Nelson SA, Todhunter RJ, Stone M, Zhang Z. Long term functional outcome of tibial tuberosity advancement vs. Tibial plateau leveling osteotomy and extracapsular repair in a heterogeneous population of dogs. Vet Surg. 2016;45(2):261-268. doi: $10.1111 /$ vsu. 12445

37. Voss K, Imhof J, Kaestner S, Montavon P. Force plate gait analysis at the walk and trot in dogs with low-grade hindlimb lameness. Vet Comp Orthop Traumatol. 2007;20(4):299-304. doi:10.1160/VCOT07-01-0008

38. Hurley CR, Hammer DL, Shott S. Progression of radiographic evidence of osteoarthritis following tibial plateau leveling osteotomy in dogs with cranial cruciate ligament rupture: 295 cases (2001-2005). $J$ Am Vet Med Assoc. 2007;230(11):1674-1679. doi:10.2460/ javma.230.11.1674

39. Au KK, Gordon-Evans WJ, Dunning D, et al. Comparison of shortand long-term function and radiographic osteoarthrosis in dogs after postoperative physical rehabilitation and tibial plateau leveling osteotomy or lateral fabellar suture stabilization. Vet Surg. 2010;39 (2):173-180. doi:10.1111/j.1532-950X.2009.00628.x

40. Moore EV, Weeren R, Paek M. Extended long-term radiographic and functional comparison of tibial plateau leveling osteotomy vs tibial tuberosity advancement for cranial cruciate ligament rupture in the dog. Vet Surg. Epub. 2019.

41. Bergh MS, Sullivan C, Ferrell CL, Troy J, Budsberg SC. Systematic review of surgical treatments for cranial cruciate ligament disease in dogs. J Am Anim Hosp Assoc. 2014;50(5):315-321. doi:10.5326/ JAAHA-MS-6356

42. Knight R, Danielski A. Long-term complication following tibial plateau levelling osteotomy in small dogs with tibial plateau angles $>30^{\circ}$. Vet Rec. 2018;182(16):461. doi:10.1136/vr.104491

43. Barnes DC, Trinterud T, Owens MR, Bush MA. Short-term outcome and complications of TPLO using anatomically contoured locking compression plates in small/medium-breed dogs with "excessive" tibial plateau angle. J Small Anim Pract. 2016;57(6):305-310. doi:10.1111/jsap.2016.57.issue-6

44. Cosenza G, Reif U, Martini FM. Tibial plateau levelling osteotomy in 69 small breed dogs using conically coupled $1.9 / 2.5 \mathrm{~mm}$ locking plates. A clinical and radiographic retrospective assessment. Vet Comp Orthop Traumatol. 2015;28(5):347-354. doi:10.3415/VCOT14-09-0135

45. Boudrieau RJ. Tibial plateau leveling osteotomy or tibial tuberosity advancement? Vet Surg. 2009;38(1):1-22. doi:10.1111/j.1532950X.2008.00439.x

46. Burns CG, Boudrieau RJ. Modified tibial tuberosity advancement procedure with tuberosity advancement in excess of $12 \mathrm{~mm}$ in four large breed dogs with cranial cruciate ligament-deficient joints. Vet Comp Orthop Traumatol. 2008;21(3):250-255. doi:10.1055/s-00371617369

47. Fitzpatrick N, Solano MA. Predictive variables for complications after TPLO with stifle inspection by arthrotomy in 1000 consecutive dogs. Vet Surg. 2010;39(4):460-474. doi:10.1111/j.1532-950X.2010. 00663.x

48. Heidorn SN, Canapp S Jr., Zink CM, Leasure CS, Carr BJ. Rate of return to agility competition for dogs with cranial cruciate ligament tears treated with tibial plateau leveling osteotomy. J Am Vet Med Assoc. 2018;253(11):1439-1444. doi:10.2460/javma.253.11.1439

49. Barnhart MD. Results of single-session bilateral tibial plateau leveling osteotomies as a treatment for bilaterally ruptured cranial cruciate ligaments in dogs: 25 cases (2000-2001). J Am Anim Hosp Assoc. 2003;39(6):573-578. doi:10.5326/0390573 
50. Cappelle KK, Barnhart MD. Short-Term complications following single-session versus staged bilateral tibial plateau levelling osteotomies stabilized with locking plates for treatment of bilateral cranial cruciate ligament disease: a retrospective study. Vet Comp Orthop Traumatol. Epub. 2019;32(6), 460-466.
51. Priddy NH, Tomlinson JL, Dodam JR, Hornbostel JE. Complications with and owner assessment of the outcome of tibial plateau leveling osteotomy for treatment of cranial cruciate ligament rupture in dogs: 193 cases (1997-2001). J Am Vet Med Assoc. 2003;222(12):1726-1732. doi:10.2460/javma.2003.222.1726

\section{Publish your work in this journal}

Veterinary Medicine: Research and Reports is an international, peerreviewed, open access journal publishing original research, case reports, editorials, reviews and commentaries on all areas of veterinary medicine. The manuscript management system is completely online and includes a very quick and fair peer-review system. Visit http://www.dovepress.com/testimonials.php to read real quotes from published authors.

Submit your manuscript here: http://www.dovepress.com/veterinary-medicine-research-and-reports-journal 